$05: 06.4$

\title{
Молекулярные магнетики - источники радиочастотного сверхизлучения при механической активации
}

\author{
() А.И. Александров ${ }^{1}$, В.Г. Шевченко ${ }^{1,9}$, И.А. Александров ${ }^{1}$, С.В. Фокин ${ }^{2}$, В.И. Овчаренко ${ }^{2}$ \\ ${ }^{1}$ Институт синтетических полимерных материалов им. Н.С. Ениколопова РАН, Москва, Россия \\ ${ }^{2}$ Международный томографический центр СО РАН, Новосибирск, Россия \\ ฯ E-mail: shev@ispm.ru
}

Поступило в Редакцию 3 июля 2020 г.

В окончательной редакции 7 сентября 2020 г.

Принято к публикации 20 сентября 2020 г.

\begin{abstract}
Установлен эффект механической активации радиочастотного сверхизлучения у композита на основе гетероспинового молекулярного магнетика и полистирольной матрицы. Механическая активация впервые проводилась в режиме реологического взрыва разгрузки.
\end{abstract}

Ключевые слова: сверхизлучение, молекулярный магнетик, композит, взрыв, разгрузка.

DOI: 10.21883/PJTF.2021.01.50453.18451

Ранее эффект механической активации радиочастотного сверхизлучения (СИ) был обнаружен в полимерных композитах, содержащих элементоорганические комплексы кобальта или марганца, которые проявляли эффект Дзялошинского-Мория [1,2]. Механическая активация проводилась при воздействии реологического взрыва исследуемого композита при быстром одноосном сжатии [1] или при воздействии импульса упругих волн, вводимого в образец по стальному волноводу [2]. Представляет научный и практический интерес реализация новых методов механической активации радиочастотного СИ для новых классов твердофазных объектов.

Цель настоящей работы - показать, что эффект механической активации радиочастотного СИ реализуется в классе твердофазных композитов на основе гетероспиновых молекулярных магнетиков, причем реализацию процесса можно проводить в режиме быстрого сброса давления, действующего на образец композита.

Для решения этих задач в работе был использован комплекс $\mathrm{Eu}(\mathrm{III})(\mathrm{SQ})_{3}$ bipy (структура приведена на рис. $1, a$, стрелками показаны спины четырех неспаренных электронов, оттенки цвета использованы для указания на электронную плотность в связях), состоящий из двух видов парамагнитных центров: первый парамагнитный ион $\mathrm{Eu}(\mathrm{III})$; второй - на лигандах на основе SQ (SQ - 3,6-ди-трет-бутилхинолятный парамагнитный лиганд); лиганд bipy (бипиридил) не парамагнитен. Комплекс $\mathrm{Eu}(\mathrm{III})(\mathrm{SQ})_{3}$ bipy имеет четыре неспаренных электрона и является гетероспиновым соединением. Методика синтеза данного комплекса приведена в работе [3], там же показано, что этот комплекс молекулярный метамагнетик, в котором наблюдается связь заряд-спин-структура.

Были синтезированы образцы композитов (композиты $K 1, K 2, K 3, K 4, K 5)$, содержащие соответственно $0.4 \cdot 10^{20}, 0.6 \cdot 10^{20}, 0.8 \cdot 10^{20}, 1.0 \cdot 10^{20}, 1.2 \cdot 10^{20}$ комплексов $\mathrm{Eu}(\mathrm{III})(\mathrm{SQ})_{3}$ bipy в $1 \mathrm{~cm}^{3}$ полистирола (PS). Об- разцы диаметром $14 \mathrm{~mm}$ и толщиной $1 \mathrm{~mm}$ были получены путем смешивания исходных компонентов с последующим прессованием смеси при $190^{\circ} \mathrm{C}$. Для получения импульсов СИ при механическом воздействии использовалась специальная ячейка высокого давления, которая помещалась в машину сжатия ИС-500 (рис. 1,b). Применялся следующий режим воздействия на образец. Вначале действует одноосная нагрузка (длится $3 \mathrm{~s}$, скорость сдавливания $1.0 \mathrm{GPa} / \mathrm{s}$ ), затем следует быстрый сброс давления при заданном давлении $3 \mathrm{GPa}$, который приводит к реологическому взрыву разгрузки (сверхбыстрому разрушению [4]). Без быстрого сброса давления ( $10 \mu \mathrm{s})$ образцы не испытывали реологического взрыва при одноосном сдавливании до $5 \mathrm{GPa}$, и соответственно импульс СИ не возникал. Схема используемой установки приведена на рис. $1, b$. Установка позволяет регистрировать переменный ток $J(t)$, генерируемый электрической составляющей $E(t)$ электромагнитного излучения, возникающего в образце. Соответственно на осциллографе фиксируется напряжение $U(t)=R J(t)$ (сопротивление $50 \Omega$ ). Импульс изменения давления на образце при разгрузке приведен на рис. $1, b$ (длится 15-20 $\mu \mathrm{s})$. Его фиксировали с помощью тензодатчика 7 (рис. $1, b)$. Импульс СИ возникал в конце фронта разгрузки спустя 7-10 $\mu$ s и длился несколько наносекунд. Эти импульсы, записанные одновременно на экране цифрового осциллографа, приведены на рис. $1, b$.

Интенсивность СИ как функция времени описывается формулой Дике [5]:

$$
I(t)=I_{0}\left(\frac{N+1}{2}\right)^{2} \operatorname{sech}^{2}\left[\frac{N+1}{2 \tau}\left(t-t_{0}\right)\right],
$$

$I(t)$ описывает импульс СИ, мощность которого достигает максимума при $t=t_{0}$. Величина максимума пропорциональна $N^{2}, t_{0}-$ время задержки импульса, $t_{0}=\tau \ln N, \tau-$ длительность импульса. Ток $J(t)$ генерируется электрической состав- 


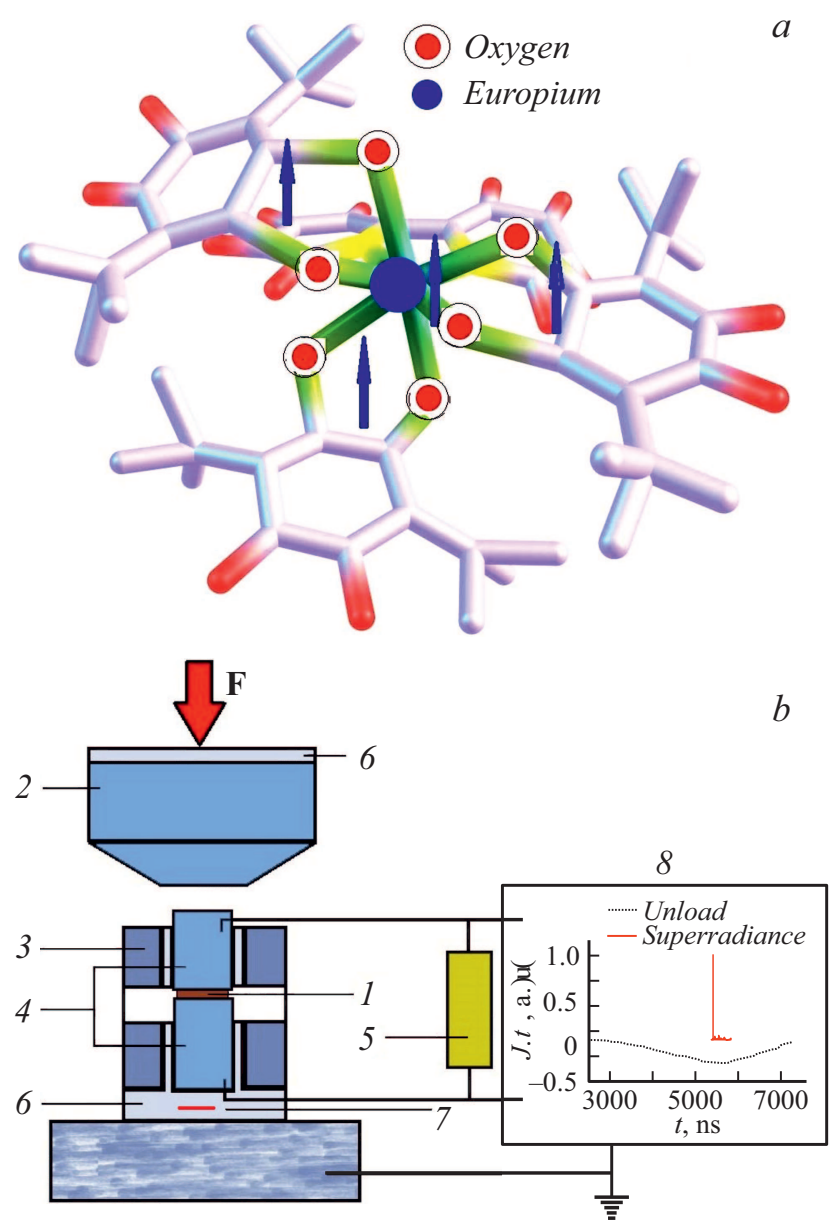

Рис. 1. $a-$ структура комплекса $\mathrm{Eu}(\mathrm{III})(\mathrm{SQ})_{3}$ bipy. $b-$ схема ячейки высокого давления: 1 - образец, 2 - наковальня Бриджмена, 3 - обойма, 4 - пуансоны, 5 - сопротивление $50 \Omega, 6$ - изоляция, 7 - тензодатчик, 8 - осциллограф с обработанными данными на экране.

ляющей $E(t)$ электромагнитного излучения, которое возникает в исследуемых образцах при механическом воздействии, поэтому $U(t) \sim J(t) \sim E(t)$. Соответственно интенсивность электромагнитного излучения $I(t) \sim[E(t)]^{2} \sim[J(t)]^{2} \sim[U(t)]^{2}$. На рис. 2, $a$ приведены характерные импульсы $I(t)$ и их аппроксимация по формуле Дике (см. (1)). Видно, что форма линии полос излучения соответствует закону (1), характерному для процессов СИ: экспоненциальному симметричному подъему и спаду. На рис. 2, $a$ анаморфозы по формуле (1) показаны пунктиром. Установлено, что интенсивность пиков $I\left(t_{0}\right)$ (при $\left.t=t_{0}\right)$ пропорциональна квадрату концентрации комплексов $N^{2}$. Это хорошо видно из рис. 2, $b$, на котором показана зависимость нормированной амплитуды $I\left(t_{0}\right)=I(N) / I\left(N_{\max }\right)$ от $N^{2}$ для комплексов $\mathrm{Eu}(\mathrm{III})(\mathrm{SQ})_{3}$ bipy. $I\left(N_{\max }\right)$ соответствует максимальному значению импульса СИ для композита $K 5$ при $3 \mathrm{GPa}$, где $N_{\max }=1.2 \cdot 10^{20}$ комплексов $\mathrm{Eu}(\mathrm{III})(\mathrm{SQ})_{3}$ bipy в $1 \mathrm{~cm}^{3}$ полистирола.
Фурье-анализ импульсов $J(t) \sim E(t)$ показал, что наблюдаемые процессы испускания СИ лежат в диапазоне частот от 0 до $300 \mathrm{MHz}$. Как видно из рис. 3, фурьеобразы сигналов $E \sim E(t) \sim J(t)$ имеют вид полосчатых спектров. Как известно, полосчатые спектры излучаются отдельными возбужденными молекулами, не связанными или слабо связанными друг с другом. Излучение вызвано как электронными переходами, так и колебательными движениями атомов в молекулах. Поэтому можно представить, что при воздействии импульса упругих волн происходят локальные размораживания молекулярной подвижности фрагментов макромолекул и дестабилизация введенных в полимерную матрицу парамагнитных комплексов, а также реализуются процессы ионизации и образование ансамбля свободных электронов. При этом возникает электромагнитное излучение, которое обычно описывается моделью гармонического осциллятора с затуханием. Поскольку в нашем случае эффект сверхизлучения реализуется многоспиновыми комплексами
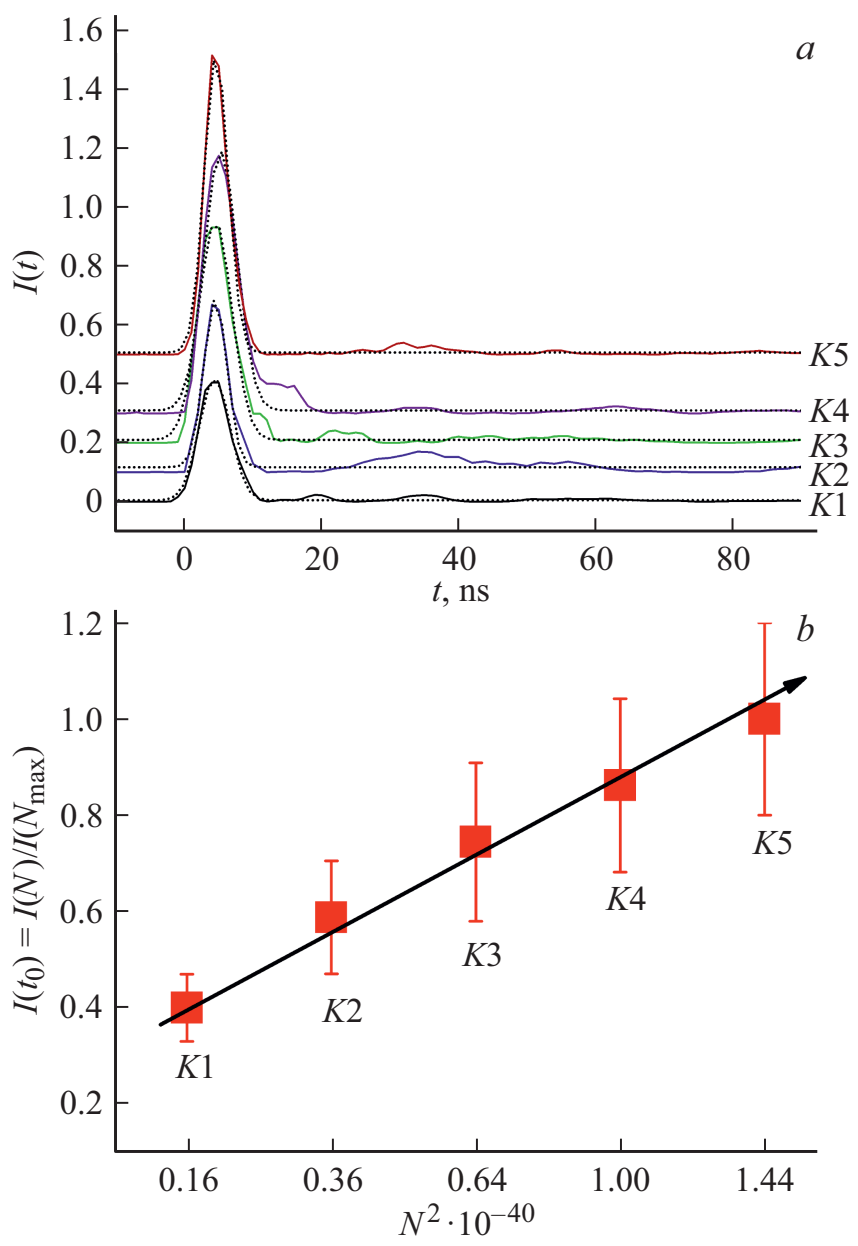

Рис. 2. $a-$ временна́я развертка нормированных сигналов $I(t) \sim[E(t)]^{2}$ для образцов композитов $K 1, K 2, K 3, K 4, K 5$. $b$ - нормированная зависимость $I\left(t_{0}\right)$ от квадрата концентрации комплексов $\mathrm{Eu}(\mathrm{III})(\mathrm{SQ})_{3}$ bipy в $1 \mathrm{~cm}^{3}$ для образцов композитов $K 1, K 2, K 3, K 4, K 5$. Нормировка проведена на максимальное значение $I\left(t_{0}\right)$ для $K 5$. 
Параметры полос $P_{1}-P_{8}$ спектра сверхизлучения для расчета по модели Лоренца

\begin{tabular}{|c|c|c|c|c|c|c|c|c|}
\hline \multirow{2}{*}{ Параметр } & \multicolumn{8}{|c|}{ Полоса спектра СИ } \\
\hline & $P_{1}$ & $P_{2}$ & $P_{3}$ & $P_{4}$ & $P_{5}$ & $P_{6}$ & $P_{7}$ & $P_{8}$ \\
\hline$\omega_{r e z, E}, \mathrm{MHz}$ & 9.50 & 27.00 & 50.00 & 75.00 & 175.00 & 204.00 & 255.00 & 306.00 \\
\hline$\omega_{r, E}, \mathrm{MHz}$ & 5.00 & 10.00 & 10.00 & 10.00 & 10.00 & 10.00 & 10.00 & 10.00 \\
\hline$\omega_{C}, \mathrm{MHz}$ & 8.07 & 25.07 & 48.98 & 71.33 & 174.71 & 203.75 & 254.80 & 305.83 \\
\hline$\varepsilon_{0}$ & 4061.53 & 1588.46 & 596.92 & 320.00 & 31.70 & 29.08 & 10.03 & 4.70 \\
\hline
\end{tabular}

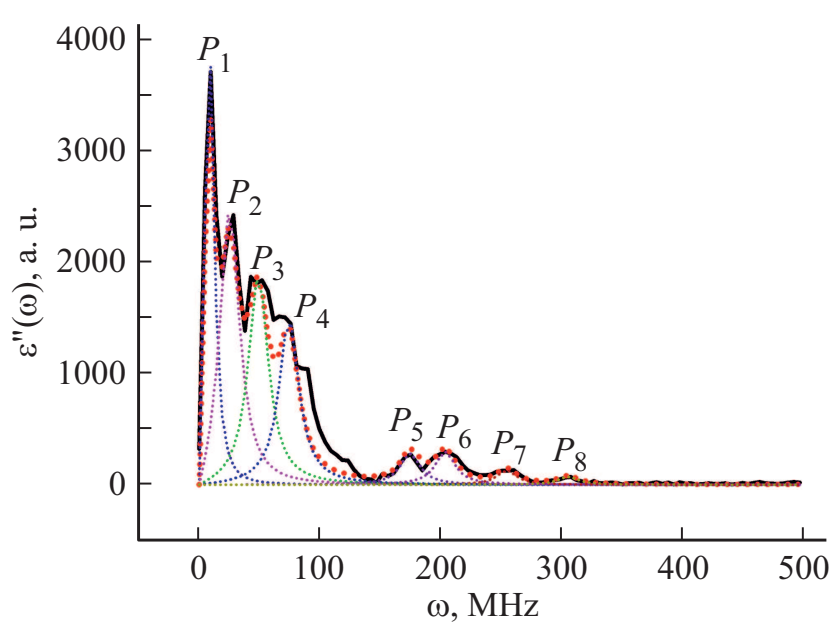

Рис. 3. Фурье-образ электрического сигнала для композита $K 5$ и полосы поглощения, полученные по методу Лоренца (их параметры приведены в таблице).

европия, можно допустить, что изменение электрического дипольного момента и изменение в структуре спиновой системы связаны между собой, т.е. электрический дипольный момент $\mathbf{D}$ связан со спиновой системой: $\mathbf{D} \propto \mathbf{R} \times\left(\mathbf{S}_{a} \times \mathbf{S}_{b}\right)[6]$, где $\mathbf{R}-$ относительное положение двух произвольно взятых спинов в комплексе $\left(\mathrm{Eu}(\mathrm{III})(\mathrm{SQ})_{3}\right.$ bipy).

Поскольку $\mathbf{R}$ изменяется во времени, изменяются векторы дипольных моментов D и векторы поляризации $\mathbf{P}$ и соответственно магнитные моменты системы $\mathbf{M}$, которые связаны со спиновым ансамблем. Динамические спектры таких систем описываются как в рамках модели Лоренца для гармонического осциллятора с затуханием [7]:

$$
\frac{\partial^{2} \mathbf{P}}{\partial t^{2}}+\omega_{r, E} \frac{\partial \mathbf{P}}{\partial t}+\omega_{r e z, E}^{2} \mathbf{P}=\omega_{C}^{2} \mathbf{E}
$$

так и в рамках модели на основе уравнения Блоха-Бломбергена [8,9]:

$$
\frac{d \mathbf{M}}{d t}=-\gamma \mathbf{M} \times \mathbf{H}+\omega_{r, H}\left[\chi_{0} \mathbf{H}-\mathbf{M}\right] .
$$

Соответственно анализировать спектры, полученные после фурье-преобразования сигналов импульсов $J(t) \sim E(t)$ для систем $\mathrm{PS}+\left(\mathrm{Eu}(\mathrm{III})(\mathrm{SQ})_{3}\right.$ bipy $)$, можно, используя приведенное для $\mathcal{E}^{\prime \prime}(\omega)$ уравнение (2) и приведенное для $\chi^{\prime \prime}(\omega)$ уравнение (3), т. е. по формулам [10-12]:

$$
\begin{aligned}
& \varepsilon^{\prime \prime}(\omega)=\varepsilon_{0} \frac{2 \omega_{r, E} \omega_{C}^{2} \omega}{\left(\omega_{r e z, E}^{2}-\omega^{2}\right)^{2}+4 \omega_{r, E}^{2} \omega^{2}}, \\
& \chi^{\prime \prime}(\omega)=\chi_{0} \frac{2 \omega_{r, H} \omega_{H} \omega^{2}}{\left(\omega_{r e z, H}^{2}-\omega^{2}\right)^{2}+4 \omega_{r, H}^{2} \omega^{2}},
\end{aligned}
$$

где $\omega_{r, E}=\Delta E[\mathrm{MHz}] / 2$ и $\omega_{r, H}=\Delta H[\mathrm{MHz}] / 2$, а $\Delta E[\mathrm{MHz}]$ и $\Delta H[\mathrm{MHz}]$ - полуширины резонансных линий. При этом $\omega_{r e z, E}^{2}=\omega_{r, E}^{2}+\omega_{C}^{2}$ и $\omega_{r e z, H}^{2}=\omega_{r, H}^{2}+\omega_{H}^{2}, \omega_{H}=\gamma H$.

Однако чтобы применить уравнения (2)-(4) для одних и тех же полос излучения, из которых формируется спектр на рис. 3, требуются дополнительные экспериментальные исследования, так как к настоящему времени нет доказательств того, что в изучаемом объекте (комплексе $\mathrm{Eu}(\mathrm{III})$ с тремя магнитными лигандами) магнитная и электронная подсистемы реализуют излучение на одних и тех же частотах. Поэтому в настоящей работе с чисто описательной целью приведен расчет полос излучения, из которых состоит суммарный спектр, только с использованием модели Лоренца.

Результаты расчетов спектра Фурье для $K 5$ при $3 \mathrm{GPa}$ по модели Лоренца для отдельных полос поглощения, из которых формируется общий спектр, представлены на рис. 3. Исходные данные для расчетов приведены в таблице. Значения $\omega_{r e z, E}$ и $\omega_{r, E}$ были взяты из „экспериментального“ фурье-спектра, а величины $\varepsilon_{0}$ выполняли роль коэффициентов пропорциональности для определения интенсивности полос $P_{1}-P_{8}$. Наблюдаемый разброс параметров для $\omega_{r e z, E}, \omega_{C}$ и $\varepsilon_{0}$ связан, вероятно, с квантовой структурой комплексов $\mathrm{Eu}(\mathrm{III})(\mathrm{SQ})_{3}$ bipy: с энергиями квантовых уровней, на которых расположены электроны, и величинами дипольных переходов между ними. Очевидно, что разработка модели, описывающей спектры сверхизлучения подобных многоспиновых комплексов, - задача для отдельного исследования.

Таким образом, в работе показано, что посредством механической активации гетероспиновых молекулярных магнетиков можно реализовать процесс радиочастотного СИ, а механическую активацию можно проводить в режиме реологического взрыва разгрузки.

\section{Финансирование работы}

Работа выполнена при поддержке Министерства науки и высшего образования РФ. 


\section{Конфликт интересов}

Авторы заявляют, что у них нет конфликта интересов.

\section{Список литературы}

[1] А.И. Александров, И.А. Александров, А.И. Прокофьев, Письма в ЖЭТФ, 97 (9), 630 (2013).

DOI: $10.7868 / \mathrm{S} 0370274 \mathrm{X} 13090105$

[2] А.И. Александров, В.Г. Шевченко, И.А. Александров, Письма в ЖТФ, 40 (7), 43 (2020).

DOI: $10.21883 /$ PJTF.2020.07.49220.18119

[3] Г.В. Романенко, С.В. Фокин, Г.А. Летягин, А.С. Богомяков, В.И. Овчаренко, ЖСХ, 60 (7), 1139 (2019).

DOI: $10.1134 / \mathrm{S} 0022476619070102$

[4] P.W. Bridgman, Rev. Mod. Phys., 18, 1 (1946).

[5] R.H. Dicke, Phys. Rev., 93, 99 (1954).

[6] T.A. Kaplan, S.D. Mahanti, Phys. Rev. B, 83, 174432 (2011). DOI: 10.1103/PhysRevB.83.174432

[7] H.A. Lorentz, Phys. Z., 498, 514 (1899).

[8] F. Bloch, Phys. Rev., 70, 460 (1946).

[9] N. Bloembergen, Phys. Rev., 78, 572 (1950).

[10] A.K. Jonscher, Dielectric relaxation in solids. (Chelsea Dielectric Press, Ltd., London, 1983).

[11] Э.Р. Блайт, Д. Блур, Электрические свойства полимеров, пер. с англ. (Физматлит, М., 2008).

[12] А.Г. Гуревич, Ферриты на сверхвысоких частотах (Физматгиз, М., 1960). 